\title{
Applications of fixed point theory to extended Nash equilibriums of nonmonetized noncooperative games on posets
}

Linsen $\mathrm{Xie}^{1}$, Jinlu Li ${ }^{2}$ and Ching-Feng Wen ${ }^{3^{*}}$

"Correspondence:

cfwen@kmu.edu.tw

${ }^{3}$ Center for Fundamental Science,

Kaohsiung Medical University, Kaohsiung, 807, Taiwan

Full list of author information is

available at the end of the article

\begin{abstract}
We say that a noncooperative game is nonmonetized if the ranges of the utilities of the players are posets. In this paper, we examine some nonmonetized

noncooperative games of which both the collection of strategies and the ranges of the utilities for the players are posets. Then we carry the concept of generalized Nash equilibriums of noncooperative games defined in (Li in J. Nonlinear Anal. Forum 18:1-11, 2013; Li and Park in Br. J. Econ. Manag. Trade 4(1), 2014) to extended Nash equilibriums of nonmonetized noncooperative games. By applying some fixed point theorems in posets and by using the order-preserving property of mappings, we prove an existence theorem of extended Nash equilibriums for nonmonetized noncooperative games.
\end{abstract}

MSC: 46B42; 47H10; 58J20; 91A06; $91 \mathrm{~A} 10$

Keywords: poset; lattice; order-preserving mapping; fixed point; nonmonetized noncooperative game; generalized Nash equilibrium; extended Nash equilibrium

\section{Introduction}

In economic theory, social science, military science, or other fields, the outcomes of some games may not be in a complete (totally) ordered set; that is, the utilities of the players may not be represented by real-valued functions, which is different from most noncooperative games in game theory. We give an example below to more precisely demonstrate our arguments.

Tanker-jet example Suppose that a military project industry produces a certain type of tankers and a certain type of fighter planes. An arbitrary considered outcome is a set of tankers and fighter planes produced by this industry. Let $U$ be the collection of all possible outcomes. Assume that the combat effectiveness of the tankers and the fighter planes is not comparable. Denote an arbitrary element in $U$ by $(x, y)$ where $x, y$ are the numbers of tankers and fighter planes in this outcome, respectively. Then, based on the incomparable capability of the outcomes, the non-rational preference relation on $U$ is defined by

$$
\left(x_{1}, y_{1}\right) \geq\left(x_{2}, y_{2}\right) \quad \text { if and only if } \quad x_{1} \geq x_{2} \quad \text { and } \quad y_{1} \geq y_{2}, \quad \forall\left(x_{1}, y_{1}\right),\left(x_{2}, y_{2}\right) \in U
$$

( 2013 Xie et al.; licensee Springer. This is an Open Access article distributed under the terms of the Creative Commons Attribution License (http://creativecommons.org/licenses/by/2.0), which permits unrestricted use, distribution, and reproduction in any medium, provided the original work is properly cited. 
This preference relation $\succeq$ on $U$ is not a complete order, which is a partial order on $U$. In case the budget of this military industry is limited (this is always true in the real world), the decision makers of this industry would seek the war production plan to optimize the fighting capability under some given warfare environment.

It is clear that such an optimization problem is not a normal optimization problem (with real-valued functions). As a matter of fact, these optimization ideas, introduced and studied by Giannessi [1] in 1980, are called vector optimization problems and vector variational inequalities, where the outcome space is in a finite-dimensional vector space. Since then, the theory as well as algorithms of vector variational inequalities and vector optimization have been extensively studied. See, e.g., [2-9] and the references therein.

Understanding the existence of optimization problems with respect to a partial order on a set of objects $U$ is of considerable interest for Li [10], Li and Park [11] to introduce the concepts of the generalized Nash equilibrium of nonmonetized noncooperative games, in which the utilities of the players may not be real-valued; that is, the payoffs of the players may not be represented by real-valued functions. Now we recall the concept of generalized Nash equilibrium of nonmonetized noncooperative games from $[10,11]$.

In an $n$-person nonmonetized noncooperative game $\Gamma=(N, S, P, U)$, where $\left(U, \succeq^{U}\right)$ is a lattice, a selection of strategies $\left(\bar{x}_{1}, \bar{x}_{2}, \ldots, \bar{x}_{n}\right) \in S_{1} \times S_{2} \times \cdots \times S_{n}$ is called a generalized Nash equilibrium of this game if the following order inequality holds:

$$
P_{i}\left(x_{i}, \bar{x}_{-i}\right) \preceq^{U} P_{i}\left(\bar{x}_{i}, \bar{x}_{-i}\right), \quad \forall x_{i} \in S_{i} \text { and for every } i=1,2,3, \ldots, n .
$$

Existence theorems of generalized Nash equilibriums of some nonmonetized noncooperative games are provided in $[10,11]$. In [10] the space of outcomes is a Banach lattice, and in [11] it is just a lattice, which is not required to be equipped with neither topological structure nor algebraic structure.

In this paper, we extend the concept of generalized Nash equilibrium to broader classes as follows: a selection of strategies $\left(\bar{x}_{1}, \bar{x}_{2}, \ldots, \bar{x}_{n}\right) \in S_{1} \times S_{2} \times \cdots \times S_{n}$ is called an extended Nash equilibrium of a nonmonetized noncooperative game $\Gamma=(N, S, P, U)$ if the following order inequality holds:

$$
P_{i}\left(x_{i}, \bar{x}_{-i}\right) \nsucc^{U} P_{i}\left(\bar{x}_{i}, \bar{x}_{-i}\right), \quad \forall x_{i} \in S_{i} \text { and for every } i=1,2,3, \ldots, n .
$$

It is clear that in both pure mathematical theory and real applications the order inequality (ii) is more general than the order inequality (i). In Section 3, we prove an existence theorem of extended Nash equilibriums of some nonmonetized noncooperative games.

\section{Preliminaries}

For the readers' convenience, in this section, we recall some concepts and properties of posets and lattices (see, e.g., [7, 12-15]). Here we closely follow the notations from [7] and [15].

Let $P=(P, \succeq)$ be a poset. An element $u$ of $P$ is called an upper bound of a subset $A$ of $P$ if $x \preceq u$ for each $x \in A$. If $u \in A$, then $u$ is called the greatest element of $A$ and is denoted by $u=\max A$. If the set of all upper bounds of $A$ has the smallest element, we call it the supremum of $A$ and denote it by $\sup A$ or $\vee A$. An element $y$ is called a maximal element of $A$ if $y \in A$ and if $z \in A$ and $y \preceq z$ imply that $y=z$. A lower bound of $A$, the smallest 
element $\min A$ of $A$, the infimum of $A, \inf A=\wedge A$ and a minimal element of $A$ can be defined similarly.

A poset $P=(P, \succeq)$ is called a lattice if $\inf \{x, y\}$ and $\sup \{x, y\}$ exist for all $x, y \in P$. Define $\inf \{x, y\}=x \wedge y$ and $\sup \{x, y\}=x \vee y$. A subset $C$ of a poset $P=(P, \succeq)$ is called a chain if $x \preceq y$ or $y \preceq x$ for all $x, y \in C$.

Definition 2.1 A poset $P=(P, \succeq)$ is said to be

(i) inductive if every chain in $P$ has an upper bound in $P$;

(ii) inversely inductive if every chain in $P$ has a lower bound in $P$;

(iii) bi-inductive whenever it is both inductive and inversely inductive.

Definition 2.2 A poset $P=(P, \succeq)$ is said to be

(i) strongly inductive whenever for every chain $C$ in $P$, the supremum of $C, \sup C$, exists in $P$;

(ii) strongly inversely inductive whenever for every chain $C$ in $P$, the infimum of $C$, $\inf C$, exists in $P$;

(iii) strongly bi-inductive whenever it is both strongly inductive and strongly inversely inductive.

Extension of Zorn's lemma Every bi-inductive poset has both maximal and minimal elements.

Let $P=(P, \succeq)$ be a poset. For any $z, w \in P$, we define the order intervals:

$$
\begin{aligned}
& {[z)=\{x \in P: x \succeq z\}, \quad(w]=\{x \in P: x \preceq w\} \quad \text { and }} \\
& {[z, w]=[z) \cap(w]=\{x \in P: z \preceq x \preceq w\} .}
\end{aligned}
$$

Given posets $\left(X, \succeq^{X}\right)$ and $\left(U, \succeq^{U}\right)$, we say that a mapping $F: X \rightarrow 2^{U} \backslash \emptyset$ is order increasing upward if $x \preceq^{X} y$ in $X$ and $z \in F(x)$ imply that $[z) \cap F(y)$ is nonempty, that is, if $x \preceq^{X} y$ in $X$ and $z \in F(x)$, then there is $w \in F(y)$ such that $z \preceq^{U} w$. The mapping $F$ is order increasing downward if $x \preceq^{X} y$ in $X$ and $w \in F(y)$ imply that $(w] \cap F(x)$ is nonempty. $F$ is said to be order increasing whenever $F$ is both order increasing upward and downward.

As a special case, a single-valued mapping $F$ from a poset $\left(X, \succeq^{X}\right)$ to the poset $\left(U, \succeq^{U}\right)$ is said to be order increasing whenever $x \preceq^{X} y$ implies $F(x) \preceq^{U} F(x)$. An increasing mapping $F: X \rightarrow U$ is said to be strictly order increasing whenever $x \prec^{X} y$ implies $F(x) \prec^{U} F(x)$.

A nonempty subset $A$ of a subset $Y$ of a poset $P=(P, \succeq)$ is said to be order compact upward in $Y$ if for every chain $C$ of $Y$ that has a supremum in $P$, the intersection $\bigcap\{[y) \cap A: y \in C\}$ is nonempty whenever $[y] \cap A$ is nonempty for every $y \in C$. The set $A$ is order compact downward in $Y$ if for every chain $C$ of $Y$ that has the infimum in $P$, the intersection $\bigcap\{(y] \cap A: y \in C\}$ is nonempty whenever $(y] \cap A$ is nonempty for every $y \in C$. If $A$ is both order compact upward and order compact downward in $Y$, then $A$ is said to be order compact in $Y$.

Let $A$ be a subset of a poset $P=(P, \succeq)$. An element $c \in P$ is called a sup-center of $A$ in $P$ if $c \vee x$ exists in $P$ for each $x \in A$. If $c \wedge x$ exists in $P$ for each $x \in A$, then $c$ is called an inf-center of $A$ in $P$. If $c$ is both a sup-center and an inf-center of $A$ in $P$, then $c$ is called an order center of $A$ in $P$. In particular, if $A=P$, then $c$ is simply called a sup-center or an inf-center of $P$, respectively. 
Let $A$ be a nonempty subset of a poset $P=(P, \succeq)$. The set $\operatorname{ocl}(A)$ is the collection of all possible supremums and infimums of chains of $A$, which is called the order closure of $A$. If $A=\operatorname{ocl}(A)$, then $A$ is said to be order closed.

Remark 2.3 Every nonempty strongly bi-inductive subset of a poset $P=(P, \succeq)$ is order closed.

Now we recall a fixed point theorem on posets from [16]. It will be used in the proof of the existence of extended Nash equilibrium of nonmonetized noncooperative games in Section 3.

Theorem 2.4 [16, Theorem 2.12] Let $P=(P, \succeq)$ be a poset. Assume that a set-valued mapping $F: P \rightarrow 2^{P} \backslash \emptyset$ is order increasing, and that its values are order compact in $F(P)$. If chains of $F(P)$ have supremums and infimums (in $P)$, and if $\operatorname{ocl}(F(P))$ has a sup-center or an inf-center in $P$, then $F$ has minimal and maximal fixed points.

\section{Nonmonetized noncooperative games on posets}

In the section of Introduction, we described the motivations to extend the noncooperative games and the concept of generalized Nash equilibrium to nonmonetized noncooperative games. In this section, we give some definitions for these extensions, which are the generalized notions of Nash equilibriums defined and studied in games theory (see, e.g., $[16-21])$.

Definition 3.1 Let $n$ be a positive integer greater than 1 . An $n$-person nonmonetized noncooperative game consists of the following elements:

(1) the set of $n$ players, which is denoted by $N=\{1,2,3, \ldots, n\}$;

(2) the collection of $n$ strategy sets $S=\left\{S_{1}, S_{2}, \ldots, S_{n}\right\}$, for the $n$ players respectively, which is also written as $S=S_{1} \times S_{2} \times \cdots \times S_{n}$;

(3) the outcome space $\left(U\right.$; $\left.\succeq^{U}\right)$ that is a poset;

(4) the $n$ utilities functions (payoff mappings) $P_{1}, P_{2}, \ldots, P_{n}$, where $P_{i}$ is the utility function for player $i$ that is a mapping from $S_{1} \times S_{2} \times \cdots \times S_{n}$ to the poset $\left(U ; \succeq^{U}\right)$ for $i=1,2,3, \ldots, n$. We define $P=\left\{P_{1}, P_{2}, \ldots, P_{n}\right\}$.

This game is denoted by $\Gamma=(N, S, P, U)$.

In an $n$-person nonmonetized noncooperative game $\Gamma=(N, S, P, U)$, when all the $n$ players $1,2,3, \ldots, n$ simultaneously and independently choose their own strategies $x_{1}, x_{2}, \ldots, x_{n}$ to play, where $x_{i} \in S_{i}$, for $i=1,2,3, \ldots, n$, then player $i$ will receive his utility (payoff) $P_{i}\left(x_{1}, x_{2}, \ldots, x_{n}\right) \in U$. For any $x=\left(x_{1}, x_{2}, \ldots, x_{n}\right) \in S$, and for every given $i=1,2,3, \ldots, n$, as usual, we define

$$
x_{-i}:=\left(x_{1}, x_{2}, \ldots, x_{i-1}, x_{i+1}, \ldots, x_{n}\right) \quad \text { and } \quad S_{-i}:=S_{1} \times S_{2} \times \cdots \times S_{i-1} \times S_{i+1} \times \cdots \times S_{n} \text {. }
$$

Then $x_{i-1} \in S_{i-1}$ and $x$ can be simply written as $x=\left(x_{i}, x_{i-1}\right)$. Moreover, we define

$$
P_{i}\left(S_{i}, x_{-i}\right)=\left\{P_{i}\left(t_{i}, x_{-i}\right): t_{i} \in S_{i}\right\}
$$

Now we extend the concept of Nash equilibrium of noncooperative games and generalized Nash equilibrium of nonmonetized noncooperative games to the extended Nash equilibrium of nonmonetized noncooperative games. 
Definition 3.2 In an $n$-person nonmonetized noncooperative game $\Gamma=(N, S, P, U)$, a selection of strategies $\left(\tilde{x}_{1}, \tilde{x}_{2}, \ldots, \tilde{x}_{n}\right) \in S_{1} \times S_{2} \times \cdots \times S_{n}$ is called an extended Nash equilibrium of this game if, for every $i=1,2,3, \ldots, n$, the following order inequality holds:

$$
P_{i}\left(x_{i}, \tilde{x}_{-i}\right) \nsucc^{U} P_{i}\left(\tilde{x}_{i}, \tilde{x}_{-i}\right), \quad \forall x_{i} \in S_{i} .
$$

Lemma 3.3 Let $\left(S_{i}, \succeq_{i}\right)$ be a poset for every $i=1,2, \ldots, n$. Let $S=S_{1} \times S_{2} \times \cdots \times S_{n}$ be the Cartesian product space of $S_{1}, S_{2}, \ldots, S_{n}$, and let $\succeq^{S}$ be the coordinate partial order on $S$ induced by the partial orders $\succeq_{i}$. That is, for any $x, y \in S$ with $x=\left(x_{1}, x_{2}, \ldots, x_{n}\right)$ and $y=\left(y_{1}, y_{2}, \ldots, y_{n}\right)$,

$$
x \succeq^{S} y \text { if and only if } x_{i} \succeq_{i} y_{i}, \quad \forall i=1,2, \ldots, n
$$

Then $\left(S, \succeq^{S}\right)$ is a poset. Furthermore, if every $\left(S_{i}, \succeq_{i}\right)$ is (strongly) inductive, then $\left(S, \succeq^{S}\right)$ is also (strongly) inductive. If every $\left(S_{i}, \succeq_{i}\right)$ is (strongly) bi-inductive, then $\left(S, \succeq^{S}\right)$ is also (strongly) bi-inductive.

Proof The proof is straightforward and is omitted here.

The following theorem is the main result of this paper. It provides some conditions for the existence of the extended Nash equilibrium of nonmonetized noncooperative games.

Theorem 3.4 Let $\Gamma=(N, S, P, U)$ be an n-person nonmonetized noncooperative game. If for every player $i=1,2,3, \ldots, n$, his strategy set $\left(S_{i}, \succeq_{i}\right)$ satisfies:

(i) $\left(S_{i}, \succeq_{i}\right)$ is a strongly inversely inductive poset;

(ii) $\left(S_{i}, \succeq_{i}\right.$ ) has a sup-center (or an inf-center simultaneously for all $i$ ); and his utility function $P_{i}$ satisfies the following conditions:

(1) $P_{i}: S \rightarrow U$ is (single-valued) order increasing with respect to the product order $\succeq^{S}$;

(2) for any fixed $x_{-i} \in S_{-i}, P_{i}\left(S_{i}, x_{-i}\right)$ is an inductive subset of $\left(U, \succeq^{U}\right)$;

(3) for any fixed $x_{-i} \in S_{-i}$, and for any $u \in P_{i}\left(S_{i}, x_{-i}\right)$, the inverse image $\left\{z_{i} \in S_{i}: P_{i}\left(z_{i}, x_{-i}\right)=u\right\}$ is a strongly bi-inductive subset of $S_{i}$;

(4) for any $\left(x_{i}, x_{-i}\right),\left(y_{i}, y_{-i}\right) \in S$ satisfying $\left(x_{i}, x_{-i}\right) \preceq^{S}\left(y_{i}, y_{-i}\right)$, the maximal elements have the following monotone properties:

(a) if $P_{i}\left(z_{i}, x_{-i}\right)$ is a maximal element of $P_{i}\left(S_{i}, x_{-i}\right)$, then there is $w_{i} \in S_{i}$ with $z_{i} \preceq_{i} w_{i}$ such that $P_{i}\left(w_{i}, y_{-i}\right)$ is a maximal element of $P_{i}\left(S_{i}, y_{-i}\right)$;

(b) if $P_{i}\left(w_{i}, y_{-i}\right)$ is a maximal element of $P_{i}\left(S_{i}, y_{-i}\right)$, then there is $z_{i} \in S_{i}$ with $z_{i} \preceq_{i} w_{i}$ such that $P_{i}\left(z_{i}, x_{-i}\right)$ is a maximal element of $P_{i}\left(S_{i}, x_{-i}\right)$.

Then this nonmonetized noncooperative game $\Gamma$ has an extended Nash equilibrium. Furthermore, this nonmonetized noncooperative game $\Gamma$ has minimal and maximal (with respect to the product lattice order $\succeq^{S}$ ) extended Nash equilibriums.

Proof For every $i=1,2, \ldots, n$, since $\left(S_{i}, \succeq_{i}\right)$ is a poset, then from Lemma 3.3, $\left(S, \succeq^{S}\right)$ is also a poset equipped with the product order $\succeq^{S}$. For every fixed $i=1,2,3, \ldots, n$, define a setvalued mapping $T_{i}: S \rightarrow 2^{S_{i}} \backslash \emptyset$ by

$$
\begin{aligned}
T_{i}(x) & =\left\{z_{i} \in S_{i}: P_{i}\left(z_{i}, x_{-i}\right) \text { is a maximal element of } P_{i}\left(S_{i}, x_{-i}\right)\right\}, \\
\forall x & =\left(x_{1}, x_{2}, \ldots, x_{n}\right) \in S .
\end{aligned}
$$


From condition (2) of this theorem, for every fixed element $x_{-i} \in S_{-i}, P_{i}\left(S_{i}, x_{-i}\right)$ is an inductive subset of $U$. Then, applying the extension of Zorn's lemma, the range $P_{i}\left(S_{i}, x_{-i}\right)$ has a maximal element; and hence $T_{i}(x)$ is a nonempty subset of $S_{i}$. Furthermore, we claim that

$T_{i}(x)$ is a nonempty strongly bi-inductive subset of $S_{i}, \quad \forall x=\left(x_{1}, x_{2}, \ldots, x_{n}\right) \in S$.

In fact, pick an arbitrary chain $C_{i}=\left\{z_{i}: z_{i} \in C_{i}\right\}$ in $T_{i}(x) \subseteq S_{i}$. Then the order increasing property of $P_{i}$ implies that $\left\{P_{i}\left(z_{i}, x_{-i}\right): z_{i} \in C_{i}\right\}$ must be a chain in $P_{i}\left(S_{i}, x_{-i}\right)$. On the other hand, from (1), $\left\{P_{i}\left(z_{i}, x_{-i}\right): z_{i} \in C_{i}\right\}$ is a collection of some maximal elements of $P_{i}\left(S_{i}, x_{-i}\right)$. Its chain property implies that $\left\{P_{i}\left(z_{i}, x_{-i}\right): z_{i} \in C_{i}\right\}$ must be a singleton. So, we can assume $P_{i}\left(z_{i}, x_{-i}\right)=u$ for all $z_{i} \in C_{i}$ and for some $u \in\left(U, \succeq^{U}\right)$. Then the set (chain) $C_{i}$ is a subset of the inverse image $\left\{t_{i} \in S_{i}: P_{i}\left(t_{i}, x_{-i}\right)=u\right\}$; that is,

$$
C_{i} \subseteq\left\{t_{i} \in S_{i}: P_{i}\left(t_{i}, x_{-i}\right)=u\right\}
$$

From condition (3), $\left\{t_{i} \in S_{i}: P_{i}\left(t_{i}, x_{-i}\right)=u\right\}$ is a strongly bi-inductive subset of $S_{i}$. It implies that the chain $C_{i}$ has both supremum and infimum in the inverse image $\left\{t_{i} \in S_{i}: P_{i}\left(t_{i}, x_{-i}\right)=\right.$ $u$, which is contained in $T_{i}(x)$. They clearly are the supremum and infimum of this chain $C_{i}$ in $T_{i}(x)$, respectively. It proves claim (2).

Define the product mapping $T: S \rightarrow 2^{S} \backslash \emptyset$ of the set-valued mappings $T_{1}, T_{2}, \ldots$, and $T_{n}$ by

$$
T(x)=T_{1}(x) \times T_{2}(x) \times \cdots \times T_{n}(x), \quad \forall x \in S .
$$

From (2) and applying Lemma 3.3, we obtain that $T(x)$ is a nonempty strongly bi-inductive subset of $\left(S, \succeq^{U}\right)$ for every $x=\left(x_{1}, x_{2}, \ldots, x_{n}\right) \in S$.

In order to apply Theorem 2.12 in [16] to show the existence of a fixed point for this mapping $T: S \rightarrow 2^{S} \backslash \emptyset$, we have to show that the set-valued mapping $T$ is increasing, that its values are order compact in $T(S)$, that chains of $T(S)$ have supremums and infimums, and that $\operatorname{ocl}(T(S))$ has a sup-center or an inf-center in $S$.

Now we show that $T: S \rightarrow S$ is an order increasing set-valued mapping with respect to the product lattice order $\succeq^{S}$. At first, we prove the order increasing upward property for $T$. For any given $x, y \in S$, with $x \preceq^{S} y$, and for any given $z \in T(x)$, we need to show that there is $w \in T(y)$ such that $z \preceq^{S} w$. We write $z=\left(z_{1}, z_{2}, \ldots, z_{n}\right)$. The definition of the product mapping $T$ and the hypothesis $z \in T(x)$ imply $z_{i} \in T_{i}(x)$; that is,

$$
P_{i}\left(z_{i}, x_{-i}\right) \text { is a maximal element of } P_{i}\left(S_{i}, x_{-i}\right) \text { for every } i=1,2, \ldots, n \text {. }
$$

Applying part (a) in condition (4) of this theorem, and from $\left(x_{i}, x_{-i}\right) \preceq^{S}\left(y_{i}, y_{-i}\right)$, there is $w_{i} \in S_{i}$ such that

$$
w_{i} \succeq_{i} z_{i} \text { for } i=1,2,3, \ldots, n,
$$

and $P_{i}\left(w_{i}, y_{-i}\right)$ is a maximal element of $P_{i}\left(S_{i}, y_{-i}\right)$; that is,

$w_{i} \in T_{i}(y)$ for $i=1,2,3, \ldots, n$. 
Let $w=\left(w_{1}, w_{2}, \ldots, w_{n}\right)$. By combining (3) and (4), we obtain $z \preceq^{S} w$ and $w \in T(y)$. It proves that $T$ is an order increasing upward mapping on $S$.

Secondly, we prove the order increasing downward property for $T$. For any given $x, y \in$ $S$, with $x \preceq^{S} y$, and for any $w \in T(y)$, we need to show that there is $z \in T(x)$ such that $z \preceq^{S} w$. Write $w=\left(w_{1}, w_{2}, \ldots, w_{n}\right)$. The hypothesis $w \in T(y)$ implies $w_{i} \in T_{i}(y)$ for every $i=1,2, \ldots, n$. That is, $P_{i}\left(w_{i}, y_{-i}\right)$ is a maximal element of $P_{i}\left(S_{i}, y_{-i}\right)$. Applying part (b) of condition (4) of this theorem, and from $\left(w_{i}, x_{-i}\right) \preceq^{S}\left(w_{i}, y_{-i}\right)$ that is implied by the condition $x \preceq^{S} y$, we obtain that $P_{i}\left(w_{i}, x_{-i}\right)$ is a maximal element of $P_{i}\left(S_{i}, x_{-i}\right)$; that is, $w_{i} \in T_{i}(x)$. Notice $w_{i} \preceq_{i} w_{i}$ for every $i=1,2, \ldots, n$. It implies $w \in T(x)$; and hence $T$ is order increasing downward. Thus $T$ is an order increasing set-valued mapping.

Next we show that $T: S \rightarrow S$ has order compact values in $T(S)$. That is, we need to show that for every $x \in S, T(x)$ is both order compact upward and order compact downward in $T(S)$. At first we show the order compact upward property of the values of $T$. Take an arbitrary chain $C$ in $T(S)$ that has a supremum in $S$, which satisfies that

$$
[y) \cap T(x) \neq \emptyset \quad \text { for every } y \in C .
$$

(Note that the condition that the given chain $C$ in $T(S)$ has a supremum in $S$ is not used in the following proof of the order compact upward property of the values of $T$.) Define $y=\left(y_{1}, y_{2}, \ldots, y_{n}\right)$. Then

$$
[y) \cap T(x)=\left(\left[y_{1}\right) \cap T_{1}(x),\left[y_{2}\right) \cap T_{2}(x), \ldots,\left[y_{n}\right) \cap T_{n}(x)\right) .
$$

Since $C$ is a chain in $T(S)$ (which has a supremum in $S$ ), the set $\left\{y_{i}: y \in C\right\}$ is a chain in $T_{i}(S)$ (and it also has a supremum in $S_{i}$ ). Hypothesis (5) and equality (6) imply

$$
\left[y_{i}\right) \cap T_{i}(x) \neq \emptyset \quad \text { for every } i=1,2,3, \ldots, n \text { and for every } y \in C \text {. }
$$

From inequality (7) we obtain that for every fixed $i=1,2,3, \ldots, n$ and for every fixed $y \in C$, there is $\mu\left(y_{i}\right) \in S_{i}$ satisfying

$$
\mu\left(y_{i}\right) \in T_{i}(x) \subseteq S_{i} \quad \text { and } \quad y_{i} \preceq_{i} \mu\left(y_{i}\right) .
$$

(Where $\mu$ can be considered as a function of two variables $y$ and $i$ defined on $C \times N$ satisfying $\mu:\{y\} \times N \rightarrow S$ for every fixed $y \in C$ and $\mu: C \times\{i\} \rightarrow S_{i}$ for every fixed $i \in N$.) So, for every fixed $i$, when $y$ varies in the chain $C$, from (8) we obtain a subset $\left\{\mu\left(y_{i}\right): y \in\right.$ $C\} \subseteq T_{i}(x) \subseteq S_{i}$ that is a chain in $T_{i}(x)$. From (2), since $T_{i}(x)$ is strongly bi-inductive and order closed, the chain $\left\{\mu\left(y_{i}\right): y \in C\right\}$ in $T_{i}(x)$ has its supremum and infimum in $T_{i}(x)$. Let the supremum of $\left\{\mu\left(y_{i}\right): y \in C\right\}$ be denoted by

$$
q_{i}=\bigvee\left\{\mu\left(y_{i}\right): y \in C\right\} \in T_{i}(x) \text { for every fixed } i=1,2,3, \ldots, n
$$

(Note: After taking the supremum operator with respect to $y \in C, q_{i}$ only depends on $i$, and it does not depend on $y$ in $C$.) By combining (8) and (9), for every fixed $i=1,2,3, \ldots, n$, we have

$$
y_{i} \preceq_{i} q_{i} \text { and } \quad q_{i} \in T_{i}(x) \quad \text { for all } y \in C \text {, }
$$


which implies

$$
q_{i} \in \bigcap\left\{\left[y_{i}\right) \cap T_{i}(x): y \in C\right\} \quad \text { for every fixed } i=1,2,3, \ldots, n \text {. }
$$

Let $q=\left(q_{1}, q_{2}, \ldots, q_{n}\right)$. From (6) and (10), we obtain $q \in \bigcap\{[y) \cap T(x): y \in C\}$. That is,

$$
\bigcap\{[y) \cap T(x): y \in C\} \neq \emptyset .
$$

Hence, $T(x)$ is order compact upward in $T(S)$.

By the strongly bi-inductive property (2), very similarly to the above proof, we can show that for every $x \in S, T(x)$ is order compact downward in $T(S)$. Hence $T(x)$ is order compact in $T(S)$.

From (2), we see that for every $x \in S, T(x)$ is a strongly bi-inductive subset of $S$. Then we show that $T(S)$ is a strongly bi-inductive subset of $S$. It is equivalent to that every chain of $T(S)$ has both supremum and infimum in $S$ (not necessarily to be in $T(S)$ ). Suppose that

$$
C=\left\{z^{\lambda}=\left(z_{1}^{\lambda}, z_{2}^{\lambda}, \ldots, z_{n}^{\lambda}\right): \lambda \in \Lambda\right\} \subseteq T(S)
$$

is a chain in $T(S)$ with index set $\Lambda$. Take a fixed $\gamma \in \Lambda$; that is, a fixed $z^{\gamma} \in C$, there is $x^{\gamma}=\left(x_{1}^{\gamma}, x_{2}^{\gamma}, \ldots, x_{n}^{\gamma}\right) \in S$ such that $z^{\gamma} \in T\left(x^{\gamma}\right)=T_{1}\left(x^{\gamma}\right) \times T_{2}\left(x^{\gamma}\right) \times \cdots \times T_{n}\left(x^{\gamma}\right)$. Then, for $i=1,2,3, \ldots, n$, it implies $z_{i}^{\gamma} \in T_{i}\left(x^{\gamma}\right)$; that is,

$$
P_{i}\left(z_{i}^{\gamma}, x_{-i}^{\gamma}\right) \text { is a maximal element of } P_{i}\left(S_{i}, x_{-i}^{\gamma}\right) .
$$

For any $z^{\lambda} \in C$ with $z^{\lambda} \succeq^{S} z^{\gamma}$, we have $z_{i}^{\lambda} \succeq_{i} z_{i}^{\gamma}$, that implies $\left(z_{i}^{\lambda}, x_{-i}^{\gamma}\right) \succeq^{S}\left(z_{i}^{\gamma}, x_{-i}^{\gamma}\right)$ for $i=$ $1,2,3, \ldots, n$. From the order increasing property of $P_{i}$, we get

$$
P_{i}\left(z_{i}^{\lambda}, x_{-i}^{\gamma}\right) \succeq^{u} P_{i}\left(z_{i}^{\gamma}, x_{-i}^{\gamma}\right) \quad \text { for } i=1,2,3, \ldots, n \text {. }
$$

Combining (11) and (12), and from $z_{i}^{\lambda} \in S_{i}$, we obtain $P_{i}\left(z_{i}^{\lambda}, x_{-i}^{\gamma}\right)=P_{i}\left(z_{i}^{\gamma}, x_{-i}^{\gamma}\right)$, which is a maximal element of $P_{i}\left(S_{i}, x_{-i}^{\gamma}\right)$ which implies that $z^{\lambda} \in C$ with $z^{\lambda} \succeq^{S} z^{\gamma}$. Then we have $z_{i}^{\lambda} \in$ $T_{i}\left(x^{\gamma}\right)$; and therefore

$$
\left\{z_{i}^{\lambda}: z^{\lambda} \in C \text { and } z^{\lambda} \succeq^{S} z^{\gamma}\right\} \subseteq T_{i}\left(x^{\gamma}\right) \text { for every fixed } i=1,2, \ldots, n
$$

Since $C$ is a chain in $T(S)$, it clearly implies that $\left\{z_{i}^{\lambda}: z^{\lambda} \in C\right.$ and $\left.z^{\lambda} \succeq^{S} z^{\gamma}\right\}$ is a chain in $T_{i}\left(x^{\gamma}\right)$. From (2), this chain $\left\{z_{i}^{\lambda}: z^{\lambda} \in C\right.$ and $\left.z^{\lambda} \succeq^{S} z^{\gamma}\right\}$ in $T_{i}\left(x^{\gamma}\right)$ has its supremum in $T_{i}\left(x^{\gamma}\right)$. Hence $\bigvee\left\{z_{i}^{\lambda}: z^{\lambda} \in C\right.$ and $\left.z^{\lambda} \succeq^{S} z^{\gamma}\right\}$ exists and it is in $T_{i}\left(x^{\gamma}\right)$, which is denoted by

$$
v_{i}=\bigvee\left\{z_{i}^{\lambda}: z^{\lambda} \in C \text { and } z^{\lambda} \succeq^{S} z^{\gamma}\right\} \in T_{i}\left(x^{\gamma}\right) .
$$

Let $v=\left(v_{1}, v_{2}, \ldots, v_{n}\right)$. Then the first part of (13) implies $v=\bigvee\left\{z^{\lambda} \in C: z^{\lambda} \succeq^{S} z^{\gamma}\right\}$. Since $C$ is a chain, and notice $z^{\lambda} \in C$, it yields that

$$
v=\bigvee\left\{z^{\lambda} \in C: z^{\lambda} \succeq^{S} z^{\gamma}\right\}=\bigvee\left\{z^{\lambda} \in C\right\}
$$


Hence $v$ is the supremum of this chain $C$ in $S$. (Furthermore, the second part of (13) implies that $v \in T\left(x^{\gamma}\right)$, that is, the supremum of this chain $C$ in $T(S)$ is in $T(S)$.)

On the other hand, for the given chain $C=\left\{z^{\lambda}=\left(z_{1}^{\lambda}, z_{2}^{\lambda}, \ldots, z_{n}^{\lambda}\right): \lambda \in \Lambda\right\} \subseteq T(S)$, we recognize that $\left\{z_{i}^{\lambda}: z^{\lambda} \in C\right\}$ is a chain in $S_{i}$. The inversely inductive property of $S_{i}$ given in condition (i) in this theorem guarantees the existence of the infimum of the chain $\left\{z_{i}^{\lambda}: z^{\lambda} \in C\right\}$ in $S_{i}$. Then

$$
b_{i}:=\bigwedge\left\{z_{i}^{\lambda}: z^{\lambda} \in C\right\}
$$

exists and is in $S_{i}$. Let $b=\left(b_{1}, b_{2}, \ldots, b_{n}\right)$. From the above definition, $b$ is the infimum of this chain $C$ in $S$. Hence, for any arbitrary chain $C$ in $T(S)$, both $\sup C$ and inf $C$ exist in $S$.

Finally, from condition (ii) of this theorem, suppose that $S_{i}$ has an inf-center for every $i=1,2, \ldots, n$. Let $c_{i}$ be an inf-center of $S_{i}$ for every $i=1,2, \ldots, n$, and let $c=\left(c_{1}, c_{2}, \ldots, c_{n}\right)$. It is clear that $c$ is an inf-center of $S$. So, $c$ is an inf-center of $\operatorname{ocl}(T(S))$ in $S$. Thus the mapping $T: S \rightarrow 2^{S} \backslash \emptyset$ satisfies all the conditions of Theorem 2.4. Hence $T$ has a fixed point $\tilde{x}=\left(\tilde{x}_{1}, \tilde{x}_{2}, \ldots, \tilde{x}_{n}\right)$ which satisfies $\tilde{x} \in T(\tilde{x})$. It implies $\tilde{x}_{i} \in T_{i}(\tilde{x})$; that is,

$$
P_{i}\left(\tilde{x}_{i}, \tilde{x}_{-i}\right) \text { is a maximal element of } P_{i}\left(S_{i}, \tilde{x}_{-i}\right) \text { for every fixed } i=1,2, \ldots, n \text {. }
$$

It is equivalent to that for every $i=1,2,3, \ldots, n$, we have

$$
P_{i}\left(t_{i}, \tilde{x}_{-i}\right) \nsucc^{U} P_{i}\left(\tilde{x}_{i}, \tilde{x}_{-i}\right) \quad \text { for all } t_{i} \in S_{i}
$$

which shows that $\tilde{x}=\left(\tilde{x}_{1}, \tilde{x}_{2}, \ldots, \tilde{x}_{n}\right)$ is an extended Nash equilibrium of this game. Furthermore, from Theorem 2.4, the mapping $T$ has minimal and maximal fixed points. Based on the above argument, they are the minimal and maximal (with respect to the product lattice order $\succeq^{S}$ ) extended Nash equilibriums for this game. This completes the proof of this theorem.

\section{Nonmonetized noncooperative games on lattices}

In this section, we consider a special case of nonmonetized noncooperative games, defined in Definition 3.1 in the last section, for the outcome space and strategy sets to be lattices. Then we examine that the generalized Nash equilibriums of nonmonetized noncooperative games defined in $[10,11]$ are the special cases of the extended Nash equilibriums defined in Definition 3.2.

Definition 4.1 [11, Definition 3.2] In an $n$-person nonmonetized noncooperative game $\Gamma=(N, S, P, U)$, with $\left(U, \succeq^{U}\right)$ to be a lattice, a selection of strategies $\left(\bar{x}_{1}, \bar{x}_{2}, \ldots, \bar{x}_{n}\right) \in S_{1} \times$ $S_{2} \times \cdots \times S_{n}$ is called a generalized Nash equilibrium of this game if the following order inequality holds

$$
P_{i}\left(x_{i}, \bar{x}_{-i}\right) \preceq^{u} P_{i}\left(\bar{x}_{i}, \bar{x}_{-i}\right)
$$

for all $x_{i} \in S_{i}$ and for every $i=1,2,3, \ldots, n$.

It is clear that any generalized Nash equilibrium of a nonmonetized noncooperative game is an extended Nash equilibrium of this game. The converse may not be true. We 
show below that Theorem 3.4 in [11] about the existence of generalized Nash equilibrium can be obtained as a corollary of Theorem 3.4 in this paper.

Corollary 4.2 [11, Theorem 3.4] Let $\Gamma=(N, S, P, U)$ be an n-person nonmonetized noncooperative game, with $\left(U, \succeq^{U}\right)$ to be a lattice. If for every player $i=1,2,3, \ldots, n$, his strategy set $\left(S_{i}, \succeq_{i}\right)$ is a strongly inversely inductive lattice and his utility function $P_{i}$ satisfies the following conditions:

(1) $P_{i}: S \rightarrow U$ is (single-valued) order increasing with respect to the product order $\succeq^{S}$;

(2) for any fixed $x_{-i} \in S_{-i}, P_{i}\left(S_{i}, x_{-i}\right)$ is an order bounded and Dedekind complete subset of $\left(U, \succeq^{U}\right)$

(3) for any fixed $x_{-i} \in S_{-i}$, and for any $u \in P_{i}\left(S_{i}, x_{-i}\right)$, the inverse image $\left\{z_{i} \in S_{i}: P_{i}\left(z_{i}, x_{-i}\right)=u\right\}$ is an order bounded and Dedekind complete subset of $S_{i}$;

(4) if $\left(w_{i}, x_{-i}\right) \preceq^{S}\left(w_{i}, y_{-i}\right)$, then for any $u \in P_{i}\left(S_{i}, x_{-i}\right) \cap\left(P_{i}\left(w_{i}, y_{-i}\right)\right]$, there is $z_{i} \in S_{i}$ such that $z_{i} \preceq_{i} w_{i}$ and $P_{i}\left(z_{i}, x_{-i}\right)=u$.

Then this nonmonetized noncooperative game $\Gamma$ has a generalized Nash equilibrium. Furthermore, $\Gamma$ has minimal and maximal (with respect to the product lattice order $\succeq^{S}$ ) generalized Nash equilibriums.

Proof One can see that any order bounded Dedekind complete subset of a lattice is a strongly bi-inductive subset of this lattice. Hence conditions (2) and (3) in this corollary imply conditions (2) and (3) in Theorem 3.4. Next, we show that conditions (1)-(4) in this corollary imply condition (4) in Theorem 3.4. As a matter of fact, under condition (2) in this corollary, we have that $P_{i}\left(w_{i}, y_{-i}\right)$ is a maximal element of $P_{i}\left(S_{i}, y_{-i}\right)$ if and only if $P_{i}\left(w_{i}, y_{-i}\right)=\bigvee_{t_{i} \in S_{i}} P_{i}\left(t_{i}, y_{-i}\right)$. Suppose $\left(w_{i}, x_{-i}\right) \preceq^{S}\left(w_{i}, y_{-i}\right)$. From condition (1), $P_{i}\left(t_{i}, x_{-i}\right) \preceq^{U} P_{i}\left(t_{i}, y_{-i}\right)$ for all $t_{i} \in S_{i}$. It implies

$$
\bigvee_{t_{i} \in S_{i}} P_{i}\left(t_{i}, x_{-i}\right) \preceq^{u} \bigvee_{t_{i} \in S_{i}} P_{i}\left(t_{i}, y_{-i}\right)=P_{i}\left(w_{i}, y_{-i}\right) .
$$

Since $P_{i}\left(S_{i}, x_{-i}\right)$ is order bounded Dedekind complete, we have

$$
\bigvee_{t_{i} \in S_{i}} P_{i}\left(t_{i}, x_{-i}\right) \in P_{i}\left(S_{i}, x_{-i}\right)
$$

Combining (14) and (15) yields

$$
\bigvee_{t_{i} \in S_{i}} P_{i}\left(t_{i}, x_{-i}\right) \in P_{i}\left(S_{i}, x_{-i}\right) \cap\left(P_{i}\left(w_{i}, y_{-i}\right)\right] .
$$

From condition (4) in this corollary, there is $z_{i} \in S_{i}$ such that $z_{i} \preceq_{i} w_{i}$ and $P_{i}\left(z_{i}, x_{-i}\right)=$ $\bigvee_{t_{i} \in S_{i}} P_{i}\left(t_{i}, x_{-i}\right)$. By applying the order increasing property of $P_{i}$, we have $P_{i}\left(w_{i}, x_{-i}\right) \succeq^{S}$ $P_{i}\left(z_{i}, x_{-i}\right)=\bigvee_{t_{i} \in S_{i}} P_{i}\left(t_{i}, x_{-i}\right)$. We then obtain

$$
P_{i}\left(w_{i}, x_{-i}\right)=\bigvee_{t_{i} \in S_{i}} P_{i}\left(t_{i}, x_{-i}\right)
$$

So, $P_{i}\left(w_{i}, x_{-i}\right)$ is also the supremum of $P_{i}\left(S_{i}, x_{-i}\right)$ in $P_{i}\left(S_{i}, x_{-i}\right)$, which is the unique maximal element of $P_{i}\left(S_{i}, x_{-i}\right)$. Taking $z_{i}=w_{i}$, then part (b) of condition (4) in Theorem 3.4 holds. 
To show that part (a) of condition (4) in Theorem 3.4 holds, for any given $x, y \in S$, with $x \preceq^{S} y$, and for any given $z_{i} \in S_{i}$ such that $P_{i}\left(z_{i}, x_{-i}\right)$ is the supremum of $P_{i}\left(S_{i}, x_{-i}\right)$ in $P_{i}\left(S_{i}, x_{-i}\right)$, which is the unique maximal element of $P_{i}\left(S_{i}, x_{-i}\right)$; that is,

$$
P_{i}\left(z_{i}, x_{-i}\right)=\bigvee_{t_{i} \in S_{i}} P_{i}\left(t_{i}, x_{-i}\right) \quad \text { for every } i=1,2, \ldots, n .
$$

For any fixed $t_{i} \in S_{i}$, the condition $x \preceq^{S} y$ clearly implies $\left(t_{i}, x_{-i}\right) \preceq^{S}\left(t_{i}, y_{-i}\right)$. Then, from condition (1) of this corollary, we obtain

$$
P_{i}\left(t_{i}, x_{-i}\right) \preceq^{U} P_{i}\left(t_{i}, y_{-i}\right) \quad \text { for every } t_{i} \in S_{i},
$$

which implies

$$
\bigvee_{t_{i} \in S_{i}} P_{i}\left(t_{i}, x_{-i}\right) \preceq^{U} \bigvee_{t_{i} \in S_{i}} P_{i}\left(t_{i}, y_{-i}\right)
$$

From condition (2) of this corollary again, the range $P_{i}\left(S_{i}, y_{-i}\right)$ is an order bounded and Dedekind complete subset of $U$. Then there is $a_{i} \in S_{i}$ such that

$$
P_{i}\left(a_{i}, y_{-i}\right)=\bigvee_{t_{i} \in S_{i}} P_{i}\left(t_{i}, y_{-i}\right)
$$

Let $w_{i}=z_{i} \vee a_{i} \in S_{i}$. Then $w_{i}$ satisfies

$$
w_{i} \succeq_{i} z_{i} \text { and } w_{i} \succeq_{i} a_{i} \quad \text { for } i=1,2,3, \ldots, n .
$$

The second order inequality above implies $\left(w_{i}, y_{-i}\right) \succeq^{S}\left(a_{i}, y_{-i}\right)$. The order-increasing property of $P_{i}: S \rightarrow U$ implies

$$
P_{i}\left(w_{i}, y_{-i}\right) \succeq^{u} P_{i}\left(a_{i}, y_{-i}\right)=\bigvee_{t_{i} \in S_{i}} P_{i}\left(t_{i}, y_{-i}\right) .
$$

Since $w_{i}=z_{i} \vee a_{i} \in S_{i}$, from the above order inequality, we get $P_{i}\left(w_{i}, y_{-i}\right)=\bigvee_{t_{i} \in S_{i}} P_{i}\left(t_{i}, y_{-i}\right)$. Hence $P_{i}\left(w_{i}, y_{-i}\right)$ is the supremum of $P_{i}\left(S_{i}, y_{-i}\right)$ in $P_{i}\left(S_{i}, y_{-i}\right)$, which is the unique maximal element of $P_{i}\left(S_{i}, y_{-i}\right)$. It shows that part (a) of condition (4) in Theorem 3.4 holds. Hence condition (4) in Theorem 3.4 is satisfied.

Condition (ii) for the set of strategies $S_{i}$ in Theorem 3.4 is satisfied. Every element of $S_{i}$ is both a sup-center and an inf-center of $S_{i}$. It is because $\left(S_{i}, \succeq_{i}\right)$ is a strongly inversely inductive lattice. So, condition (i) for the set of strategies $S_{i}$ in Theorem 3.4 is also satisfied.

Thus, from Theorem 3.4, the game $\Gamma=(N, S, P, U)$ has an extended Nash equilibrium $\left(\bar{x}_{1}, \bar{x}_{2}, \ldots, \bar{x}_{n}\right) \in S_{1} \times S_{2} \times \cdots \times S_{n}$ satisfying that for every $i=1,2,3, \ldots, n$, the following order inequality holds:

$$
P_{i}\left(x_{i}, \bar{x}_{-i}\right) \chi^{U} P_{i}\left(\bar{x}_{i}, \bar{x}_{-i}\right) \quad \text { for all } x_{i} \in S_{i} .
$$

We claim that $\left(\bar{x}_{1}, \bar{x}_{2}, \ldots, \bar{x}_{n}\right)$ is a generalized Nash equilibrium of this game $\Gamma=(N, S, P, U)$. To show this claim, we need to prove that for every $i=1,2,3, \ldots, n$, the following order 
inequality holds:

$$
P_{i}\left(x_{i}, \bar{x}_{-i}\right) \preceq^{U} P_{i}\left(\bar{x}_{i}, \bar{x}_{-i}\right) \quad \text { for all } x_{i} \in S_{i} .
$$

To this end, assume, by the way of contradiction, that there is $z_{i} \in S_{i}$ such that

$$
P_{i}\left(z_{i}, \bar{x}_{-i}\right) \npreceq^{U} P_{i}\left(\bar{x}_{i}, \bar{x}_{-i}\right) .
$$

Since $P_{i}\left(S_{i}, \bar{x}_{-i}\right)$ is an order bounded Dedekind complete sub-lattice of $S_{i}$,

$$
P_{i}\left(z_{i}, \bar{x}_{-i}\right) \vee P_{i}\left(\bar{x}_{i}, \bar{x}_{-i}\right) \in P_{i}\left(S_{i}, \bar{x}_{-i}\right) .
$$

Therefore, there is $y_{i} \in S_{i}$ such that

$$
P_{i}\left(y_{i}, \bar{x}_{-i}\right)=P_{i}\left(z_{i}, \bar{x}_{-i}\right) \vee P_{i}\left(\bar{x}_{i}, \bar{x}_{-i}\right) .
$$

From (17), the supremum in (18) implies

$$
P_{i}\left(y_{i}, \bar{x}_{-i}\right) \succ^{U} P_{i}\left(\bar{x}_{i}, \bar{x}_{-i}\right) .
$$

It is a contradiction to (16) and this corollary is proved.

\section{Competing interests}

The authors declare that they have no competing interests.

\section{Authors' contributions}

All authors contributed equally to this work. All authors read and approved the final manuscript.

\section{Author details}

${ }^{1}$ Department of Mathematics, Lishui University, Lishui, Zhejiang 323000, China. ${ }^{2}$ Department of Mathematics, Shawnee State University, Portsmouth, Ohio 45662, USA. ${ }^{3}$ Center for Fundamental Science, Kaohsiung Medical University, Kaohsiung, 807, Taiwan

\section{Acknowledgements}

The first author was partially supported by National Natural Science Foundation of China (11171137). The third author was partially supported by a grant from NSC 102-2115-M-037-001.

Received: 4 June 2013 Accepted: 15 August 2013 Published: 13 September 2013

\section{References}

1. Giannessi, F: Theorems of alternative, quadratic programs and complementarity problems. In: Cottle, RW, Giannessi, F, Lions, J-L (eds.) Variational Inequalities and Complementarity Problems, pp. 151-186. Wiley, New York (1980)

2. Ansari, QH, Yao, JC: On nondifferentiable and nonconvex vector optimization problems. J. Optim. Theory Appl. 106 487-500 (2000)

3. Ansari, $Q H$, Yang, XQ, Yao, JC: Existence and duality of implicit vector variational problems. Numer. Funct. Anal. Optim. 22(7-8), 815-829 (2001)

4. Ceng, LC, Chen, GY, Huang, XX, Yao, JC: Existence theorems for generalized vector variational inequalities with pseudomonotonicity and their applications. Taiwan. J. Math. 12, 151-172 (2008)

5. Ceng, LC, Schaible, S, Yao, JC: Existence of solutions for generalized vector variational-like inequalities. J. Optim. Theory Appl. 137, 121-133 (2008)

6. Ceng, LC, Yao, JC: Approximate proximal methods in vector optimization. Eur. J. Oper. Res. 183, 1-19 (2007)

7. Chen, GY: Vector variational inequalities and its applications for multiobjective optimization. Chin. Sci. Bull. 34 969-972 (1989)

8. Chuong, TD, Mordukhovich, B, Yao, JC: Hybrid approximate proximal algorithms for efficient solutions in vector optimization. J. Nonlinear Convex Anal. 12, 257-286 (2011)

9. Chuong, TD, Yao, JC: Generalized Clarke epiderivatives of parametric vector optimization problems. J. Optim. Theory Appl. 146, 77-94 (2010) 
10. Li, JL: Applications of fixed point theory to generalized Nash-equilibriums of nonmonetized noncooperative games on Banach lattices. Nonlinear Anal. Forum 18, 1-11 (2013)

11. Li, JL, Park, S: Generalized Nash-equilibriums of non-monetized non-cooperative games on lattices. Br. J. Econ. Manag. Trade 4(1) (2014)

12. Agarwal, RP, Balej, M, O’Regan, D: A unifying approach to variational relation problems. J. Optim. Theory Appl. 154, 417-429 (2012)

13. Aliprantis, CD, Burkinshaw, O: Positive Operators. Springer, Dordrecht (2006)

14. Dunford, N, Schwartz, JT: Linear Operators. Part I. Wiley, New York (1988)

15. Ok, EA: Order Theory (forthcoming)

16. Carl, S, Heikkilä, S: Fixed Point Theory in Ordered Sets and Applications: From Differential and Integral Equations to Game Theory. Springer, New York (2010)

17. Debreu, G: Theory of Value. Wiley, New York (1959)

18. Glicksberg, I: A further generalization of the Kakutani fixed point theorem with applications to Nash equilibrium points. Proc. Am. Math. Soc. 3, 170-174 (1952)

19. Mas-Colell, A, Whinston, MD, Green, JR: Microeconomic Theory. Oxford University Press, London (1995)

20. Samuelson, P: Foundations of Economic Analysis. Harvard University Press, Cambridge (1947)

21. Von Neumann, J, Morgenstern, O: The Theory of Games and Economic Behavior. Princeton University Press, Princeton (1944)

doi:10.1186/1687-1812-2013-235

Cite this article as: Xie et al.: Applications of fixed point theory to extended Nash equilibriums of nonmonetized noncooperative games on posets. Fixed Point Theory and Applications 2013 2013:235.

\section{Submit your manuscript to a SpringerOpen ${ }^{\circ}$ journal and benefit from:}

- Convenient online submission

Rigorous peer review

- Immediate publication on acceptance

- Open access: articles freely available online

- High visibility within the field

- Retaining the copyright to your article 